\title{
Astrocyte elevated gene 1: biological functions and molecular mechanism in cancer and beyond
}

\author{
Zhe Ying ${ }^{1,3}$, Jun $\mathrm{Li}^{2,3}$ and Mengfeng $\mathrm{Li}^{1,3^{*}}$
}

\begin{abstract}
Since its discovery, nearly one decade of research on astrocyte elevated gene 1 (AEG-1) has witnessed expanding knowledge of this molecule, ranging from its role in cancer biology to molecular mechanisms underlying the biological functions. As a multifunctional oncoprotein, AEG-1 has been shown to overexpress in multiple types of human cancer, and the elevation of AEG-1 in tumor cells leads to enhanced phenotypes characteristic of malignant aggressiveness, including increased abilities to proliferate robustly, to invade surrounding tissues, to migrate, to induce neovascularization, and to enhance chemoresistance. The multifunctional role of AEG-1 in tumor development and progression has been found to be associated with several signaling cascades, namely, 1) activation of NF-kappa B, partially through direct interaction with p65; 2) PI3K/AKT signaling triggered by AEG-1 indirectly; 3) enhancement of the transcriptional activity of beta-catenin by indirect activation of MAPK and induction of LEF1; 4) regulation of mi/siRNA-mediated gene silencing by interacting with SND1; and 5) promotion of protective autophagy; in addition to possibly unknown mechanisms. Elevated AEG-1 expression is seen in nearly all tumor types, and in most cases AEG-1 positively correlates with tumor progression and poorer patient survival. Taken together, AEG-1 might represent a potential prognostic biomarker and therapeutic target.
\end{abstract}

\section{Discovery and molecule characteristics of AEG-1}

In 2002 and 2004, several groups, under different experimental settings, reported identification of the gene, first named as astrocyte elevated gene 1 (AEG-1), now having the GenBank symbol as MTDH, which stands for metadherin, for its involvement in tumor metastasis and adhesion. In an attempt to identifying molecules involved in mediating HIV-related neuron degeneration, Fisher's group, by using subtraction hybridization, found AEG-1 as a novel transcript induced by HIV-1 infection or gp120 treatment in human fetal astrocytes [1]. In another study by Brown and Ruoslahti in 2004, phage display strategy was employed in a mouse model of breast cancer metastasis, which revealed that a gene identical to AEG-1 was expressed at high levels in relation to metastasis of breast cancer, and the identified gene was named metadherin after its proposed role in promoting homing of breast cancer cells to the lungs [2]. In the same year, Britt et al. and Heidi et al., independently, identified that the same

\footnotetext{
* Correspondence: limf@mail.sysu.edu.cn

'Department of Microbiology, Zhongshan School of Medicine, Sun Yat-sen University, Guangzhou, Guangdong 510080, China

Full list of author information is available at the end of the article
}

molecule, this time designated LYRIC in both cases, was a novel component of the tight junction structure of epithelial cells [3] and an endoplasmic reticulum/nuclear envelope-associated protein [4], respectively.

While the initial discovery of AEG-1 left with an abundance of controversial understanding of its biological functions and biochemical characteristics, which remains elusive to date, a few features of AEG-1 have been recognized with certain consensus. In human, AEG-1 represents a protein of 582 amino acids, and the amino acid sequences are highly conserved across vertebrates. Despite its conservativeness, however, the threedimensional structure of AEG-1 has not been solved, and the functional domains of the protein are not clearly defined. To understand the biological functions of AEG-1 in cells, however, its localization has been investigated. In previously published studies, the intracellular localization of AEG-1 appeared to be variable and depend on the cell types examined and the imaging techniques employed. In most cases both endogenous or ectopically expressed AEG-1 is detectable in cytoplasm as well as in the nucleus by immunohistochemical and immunofluorescent staining of cultured cells or sectioned specimens of tissues [4-7]. In rat liver, however, 
AEG-1 was displayed on cell membrane [3], whereas GFP-fused AEG-1 showed stronger staining in the nucleolus $[8,9]$. Three putative NLS has been predicted in the lysine-rich regions of AEG-1 $[4,6]$. In the latter report, interestingly, the functional importance of these three regions was examined, and by using the GFP reporting system, NLS 1 or 3 and their flanking regions was shown to be able to target the reporter GFP peptide to nuclear and nucleolus [9]. Moreover, as a protein rich of the lysine residues, AEG-1 could be potential subject to post-translational ubiquitination, SUMOlation or acetylation [6]. There has been evidence indicating potential mono-ubiquitination of AEG-1 in its NLS2 sequence and the flanking regions, and ubiqutinated AEG-1 was found in the cytoplasm [9]. These observations might explain why AEG-1 of predicted $64 \mathrm{kD}$ molecule weight exhibits bands between 70-80 kD when detected by antibodies raised against various AEG-1 immunogens fragments. Nevertheless, thus far the significance of post-translational modification on the molecular function of AEG-1 remains to be understood.

\section{Elevated expression of AEG-1 in human cancers and other conditions}

Elevation of AEG-1 expression has been appreciated in a spectrum of cancer types. Breast cancer, glioma and prostate cancer were among the first tumor types in which AEG-1 upregulation was recognized $[6,7,10]$. In breast cancer, it has found that the degree of AEG-1 expression correlates with clinical staging and tumornode-metastasis (TNM) classification of the disease and might be useful as a potential prognostic marker [7]. Through copy number variation/gene expression association analysis, AEG-1 has been identified as a metastasis-associated gene whose overexpression is attributable to genomic amplification in breast cancer [11]. In esophageal squamous cell carcinoma, non-small cell lung cancer and hepatocellular carcinoma, the level of AEG-1 upregulation also correlates with clinical progression (staging) and poor prognosis of patients [12-14], further suggesting that AEG-1 might function as an oncogenic protein in various tumor types. Other tumor types in which upregulation of AEG-1 has been demonstrated include gastric cancer [15], colorectal carcinoma [16], osteosarcoma [17].

It is of note that in other conditions than cancer, altered expression of AEG-1 has also been reported. For example, an allele of rs1835740 near the AEG-1 locus is associated with migraine and increased AEG-1 expression [18]. Moreover, a recent study on the expression pattern of AEG-1 has indicated a possible role of AEG-1 in the development of brain, liver and skin [19]. In addition, AEG-1 could be induced by LPS stimulation of the U937 human promonocytic cells, suggesting a role of the molecule in toll-like receptor-mediated signaling [20].

AEG-1 overexpression in cancer has been attributed to an increase in the amount of its transcript resulting from one or both of two the following molecular mechanisms. Firstly, high AEG-1 expression in breast cancer and hepatocellular carcinoma is reportedly associated with genomic amplification of the AEG-1 locus $[11,14]$. The second mechanism, as suggested by several studies on the transcriptional regulation of the gene, is postulated based on the finding that AEG-1 mRNA is inducible by several regulatory signals. Activation of the Ras oncogene and subsequent induction of oncogenic transcription factor c-MYC leads to recruitment of cMYC to the AEG-1 promoter region, which consequently transactivates AEG-1 expression [21]. Furthermore, transcription suppressor PLZF has been found to interact with AEG-1 and to facilitate the export of PLZF from the nucleus to cytoplasm, thereby freeing a binding region in the AEG-1 promoter/enhancer from the occupancy by the suppressive PLZF, leading to increased binding of transactivators, such as c-MYC, to its own promoter [8]. This postulated model provides a rational basis for the insight that a positive feedback loop might exist to support AEG-1 overexpression in pathological conditions. Pro-inflammatory factors including TNFalpha and LPS could induce AEG-1 in both tumor and non-tumor cells $[1,20]$. While these stimuli are likely to activate inflammation-stimulated transcription factors such as NF kappa B and AP-1, the significance of AEG1 in mediating the reciprocal interaction between inflammation and tumor development/progression remains to be determined. For most tumor types, the proportion of tumor samples with AEG-1 overexpression is significantly higher than that of those harboring AEG-1 amplification. Hence, it would be of great interest to further investigate whether cells harboring AEG-1 amplification seed a microenvironment that promotes AEG-1 expression throughout tumor progression.

\section{Biological functions of AEG-1}

Interest in the effect of AEG-1 on the functions and the malignant phenotype of cancer cells is emerging as a hotspot in the field of cancer biology. Initial evidence demonstrated that overexpressing AEG-1 subjected breast cancer cells to lung homing [2], which was then further confirmed by in vivo experiments [11]. Furthermore, AEG-1 was demonstrated to promote invasion and migration of cancer cells. Emdad et al. first reported that the NF kappa B pathway was required for AEG-1induced promotion of invasion of Hela cells in vitro [5]. In parallel, we found that AEG-1 promoted the invasion of glioma cells in mouse with an in situ xenotranplantion glioma model [22] through directly targeting and 
transactivating the promoter of MMP-9 gene, a molecule broadly recognized to be a major metalloproteinase required for ECM degradation and cytokine activation of invading cells during the processes of tumor invasion and metastasis, as well as other physiological and pathological conditions [23].

Another oncologically important notion is the possible involvement of AEG-1 in establishing chemoresistance, a hallmark of tumor aggressiveness [24]. In breast cancer, elevated AEG-1 enables tumor cells to escape cell death induced by paclitaxel, doxorubicin or cisplatin [11]. In this model, AEG-1-induced chemoresistance was thought to be mediated by survival-promoting genes, most notably ALDH3A1 and c-MET. In the same study, moreover, hydrogen peroxide-induced oxidative stress could also be relieved by AEG-1 downstream gene HOMOX1 [11]. Another investigation aimed to identify regulators of 5-FU resistance gene showed that AEG-1 promoted the expression of DPYD, the rate-limiting enzyme of 5-FU degradation [25]. AEG-1 has been shown to mediate upregulation of MDR1 via the PI3K/ AKT signaling and augmentation of translation [26], a mechanism key to AEG-1-mediated efflux of doxorubicin. It is also noteworthy that AEG-1 overexpression is also associated with development of resistance in cells against other types of stress, such as hypoxia and glucose deprivation [27]. Moreover, evidence has shown that AEG-1 could trigger protective autophagy, which is a common mechanism employed by cancer cells to cope with metabolic stress. Recent studies have shown that by activating AMPK AEG-1 is able to elevate autophagy regulator ATG5 and lead to increased autophagy [28].

AEG-1 also contributes to tumor growth through proproliferative and anti-apoptotic effects. In prostate cancer [10] and breast cancer [29] elevation of AEG-1 has been clearly linked to downregulation of cell cycle inhibitors, leading to accelerated cell proliferation. Furthermore, in other models, AEG-1 has been found to be sufficient to suppress apoptosis through a systematic mechanism that upregulates apoptosis inhibitors via activation of the PI3K/Akt signaling pathway [30].

In many tumor types that display elevated AEG-1 expression, AEG-1 has been shown to enhance tumorinduced angiogenesis. In tumors formed by AEG-1transduced rat embryo fibroblasts, intensive angiogenic markers including Ang-1, HIF1 alpha and CD31 are correlated with AEG-1 upregulation. It appears that the PI3K/AKT signaling is involved in AEG-1 induced angiogenesis [31]. Interestingly, in addition to its role in promoting the pro-angiogenic activity of tumor cells, endothelial AEG-1 might also be able to enhance the capability of vascular endothelial cells to form neovascularture, as ectopic expression of AEG-1 in HUVEC cells was found to result in increased tube formation [31].
Moreover, analysis of clinical samples taken from large cohorts of patients of breast cancer showed that AEG-1 correlated positively with the VEGF level and the microvascular density [32].

\section{Activation of oncogenic signal cascades by AEG-1}

The molecular mechanisms underlying the oncogenic role of AEG-1 in human cancers has been studied during this decade. There has been a large abundance of evidence demonstrating that several major cellular signaling cascades might be associated with the ability of AEG-1 to execute the identified biological functions in various contexts. The NF kappa B pathway was first reported to be activated by AEG-1 through physical interaction with the p65 subunit of NF kappa B complex [5], with further evidence revealing that binding of AEG-1 to p65 resulted in recruitment of acetyltransferase CBP to $\mathrm{p} 65$, which might contribute to transcriptional activation [33]. It is believed that depending on the residue sets that are acetylated, the affinity with which p65 binds to I kappa B alpha can be increased or decreased, and thus the transcription activity of p65 is activated or inhibited correspondingly [34]. In the scenario of p65 activation induced by AEG-1, whether the recruitment of CBP results in acetylation of transactivating residues of p65 or alternatively other component(s) of the p65-containing complex that occupies the target promoter remains to be elucidated.

As aforementioned, in the attempt to understand the molecular mechanism that mediates the expression of AEG-1, the PI3K/AKT was identified to be required for Ras-induced AEG-1 expression [21]. Interestingly, the same study found that AKT was also a downstream mediator of the functions of AEG-1, as overexpression or silencing of AEG-1 resulted in elevation or attenuation, respectively, of the phosphorylation status of AKT $[10,29,30]$. Activation of AKT by AEG-1 led to phosphorylation of GSK3-beta and phosphorylation suppression of FOXO1/3 activity, resulting in enhanced cell survival and proliferation $[10,29,30]$. Given that PI3K/ AKT signaling represents a commonly activated oncogenic pathway in various cancers and crosstalks with multiple signaling pathways, including NF kappa B [35], it is likely that AKT activation could further enhance the activation NF kappa B induced by AEG-1. In hepatocellular carcinoma, AEG-1 has been shown to increase phosphorylation of MAPK molecules, including ERK1/2 and p38, which subsequently activates Wnt-mediated signaling and consequently leads to increased tumor angiogenesis [14].

Interaction of AEG-1 with other proteins and the subsequent signaling effects remain to be understood. While several signaling pathways involved in mediating the oncogenic functions of AEG-1 have been elucidated, 
little is known about what direct interactions occur between AEG-1 and other proteins and how they contribute to the downstream effects of AEG-1.

\section{Engagement of AEG-1 in the RNA-induced silencing complex (RISC)}

Recent reports by two independent groups have shown that AEG-1 could interact with components of the RISC complex. By employing yeast two-hybrid method, coimmunoprecipitation and mass-spectrometry, SND1 of the RISC complex was identified as a novel binding partner of AEG-1 [36,37]. SND1 was initially dentified as a transcription co-activator interacting with viral transcription factor EBNA2 [38] and as an activator of c-Myb [39] and STAT6 [40]. It was later reported to be contained in the RISC complex [41] and thus important for the editing and subsequent degradation of dsRNA [42]. Disruption of SND1 in C. elegance results in defects in the action of siRNA [41], which underscores its importance in RNAi-induced gene silencing. As AEG-1 has been demonstrated to interact with SND1 and enhance the activity of RISC [36], it increases degradation of tumor suppressor mRNAs that are target of onco-miRs [37]. What remains interesting is that AEG-1 mediated-activation of RISCs appears to be a general observation for all RISCs in cells with high AEG-1 expression, and yet it is unclear whether the net oncogenic effects of microRNAs documented in cancer cells are due to a selective interaction between AEG-1 and onco-miR-containing RISCs. Alternatively, AEG-1 may facilitate the function of all RISCs in a non-selective manner, and the oncogenic effects of microRNAs merely result from the fact that tumor cells harbor onco-miRs at higher levels than those of tumor suppressive microRNAs. Nevertheless, despite that the significance of the interactions between AEG-1 and RISC demands further investigation, the finding of AEG-1 as a component of RISC might provide new insights in how AEG-1 promotes a wide variety of oncogenic activities at a highly context-dependent fashion. To understand how AEG-1 affects RISC activity, studies on whether AEG-1 could regulate RNA recognition or cleavage activity are essential.

\section{Summary and perspectives}

Accumulating evidence has shown that AEG-1 plays remarkable roles in the regulation of various physiological and pathological processes [43]. A model presented as Figure 1 attempts to illustrate possible molecules and pathways that have been suggested by mechanistic studies to mediate the biological functions of this important protein. Although a great abundance of literature has documented an array of cellular functions associated with altered expression level of AEG-1, we are yet far

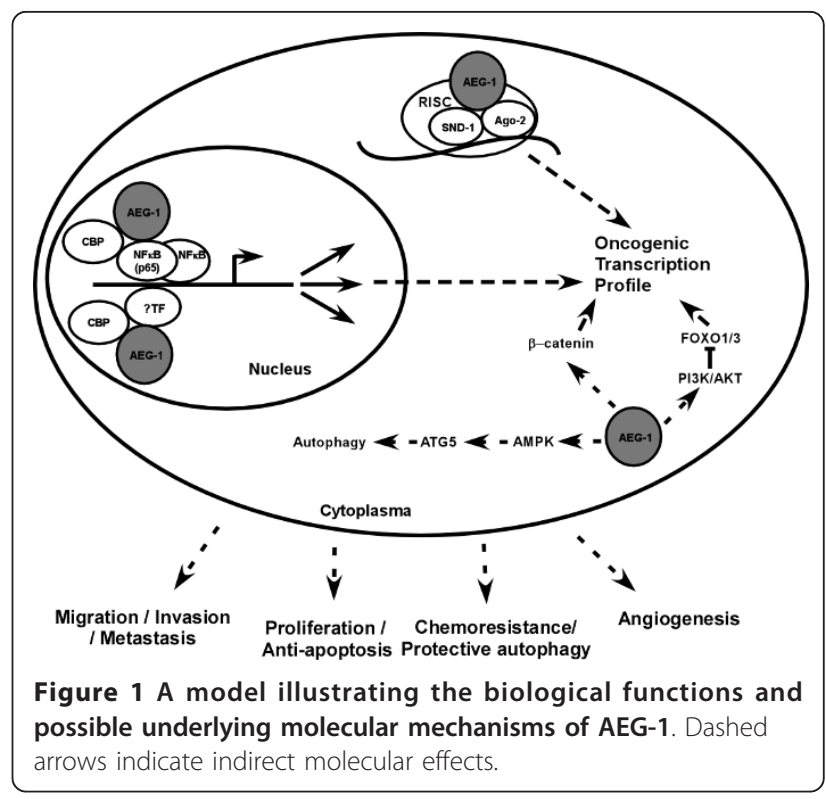

from revealing the biological significance of this molecule in physiological and pathological conditions. Experimental evidence from AEG-1 knockout or transgenic animal models would be important for further understanding of the functional significance of AEG-1 in embryonic development and diseases. Evolutionally, AEG-1 is only found in vertebrates that have complete immune systems developed, and it is noteworthy that the signaling pathways identified thus far modulated by AEG-1 have also been associated with the regulation of inflammation and immune response. It is thus of importance to further investigate the significance of aberrated AEG-1 expression in pathogenesis related to abnormal immune response or nonresolving inflammation, such as cancer, autoimmune diseases and severe immunopathological conditions caused by pathogen infection. At the molecule level, evidence is still needed to illustrate whether AEG-1 exerts its biological function by biochemically modifying its binding partners or alternatively, by acting as a scaffold of the signaling/effector complex. Moreover, the biochemical modification occurring to AEG-1 itself is also of great interest for further investigation, as such modification(s), if proved to be functionally important for AEG-1, would be potentially promising targets for optimal inhibitors or therapeutic drugs against diseases involving upregulated or overactivated AEG-1, including cancer.

AEG-1 is also under investigation and development for its potential value as a diagnostic or prognostic biomarker based on its overexpression and correlations with disease staging and outcome throughout a wide range of cancer types. Yet for the latter application, however, larger cohorts of patient studies and 
prospective investigation on the correlation of AEG-1 mRNA or protein in blood, urine and biopsy samples with clinical characteristics are urgently needed.

\section{Acknowledgements}

This work was supported by the Ministry of Science and Technology of China grant (973)2011CB11305; the national science foundation grants 30872930, 81071647

\section{Author details}

'Department of Microbiology, Zhongshan School of Medicine, Sun Yat-sen University, Guangzhou, Guangdong 510080, China. ${ }^{2}$ Department of Biochemistry, Zhongshan School of Medicine, Sun Yat-sen University, Guangzhou, Guangdong 510080, China. ${ }^{3}$ Key Laboratory of Tropical Disease Control (Sun Yat-sen University), Chinese Ministry of Education, Guangzhou 510080, China

\section{Authors' contributions}

$Z Y, J L$ and MFL wrote the review. All authors read and approved the final manuscript.

\section{Competing interests}

The authors declare that they have no competing interests.

Received: 6 August 2011 Accepted: 7 November 2011

Published: 7 November 2011

\section{References}

1. Su ZZ, Kang DC, Chen Y, Pekarskaya O, Chao W, Volsky DJ, Fisher PB: Identification and cloning of human astrocyte genes displaying elevated expression after infection with HIV-1 or exposure to HIV-1 envelope glycoprotein by rapid subtraction hybridization, RaSH. Oncogene 2002, 21:3592-3602.

2. Brown DM, Ruoslahti E: Metadherin, a cell surface protein in breast tumors that mediates lung metastasis. Cancer Cell 2004, 5:365-374.

3. Britt DE, Yang DF, Yang DQ, Flanagan D, Callanan H, Lim YP, Lin SH, Hixson DC: Identification of a novel protein, LYRIC, localized to tight junctions of polarized epithelial cells. Exp Cell Res 2004, 300:134-148.

4. Sutherland HG, Lam YW, Briers S, Lamond Al, Bickmore WA: 3D3/lyric: a novel transmembrane protein of the endoplasmic reticulum and nuclear envelope, which is also present in the nucleolus. Exp Cell Res 2004, 294:94-105.

5. Emdad L, Sarkar D, Su ZZ, Randolph A, Boukerche H, Valerie K, Fisher PB: Activation of the nuclear factor kappaB pathway by astrocyte elevated gene-1: implications for tumor progression and metastasis. Cancer Res 2006, 66:1509-1516.

6. Emdad L, Sarkar D, Su ZZ, Lee SG, Kang DC, Bruce JN, Volsky DJ, Fisher PB: Astrocyte elevated gene-1: recent insights into a novel gene involved in tumor progression, metastasis and neurodegeneration. Pharmacol Ther 2007, 114:155-170

7. Li J, Zhang N, Song LB, Liao WT, Jiang LL, Gong LY, Wu J, Yuan J, Zhang $H Z$, Zeng MS, Li M: Astrocyte elevated gene-1 is a novel prognostic marker for breast cancer progression and overall patient survival. Clin Cancer Res 2008, 14:3319-3326.

8. Thirkettle HJ, Mills IG, Whitaker HC, Neal DE: Nuclear LYRIC/AEG-1 interacts with PLZF and relieves PLZF-mediated repression. Oncogene 2009, 28:3663-3670.

9. Thirkettle HJ, Girling J, Warren AY, Mills IG, Sahadevan K, Leung H, Hamdy F, Whitaker HC, Neal DE: LYRIC/AEG-1 is targeted to different subcellular compartments by ubiquitinylation and intrinsic nuclear localization signals. Clin Cancer Res 2009, 15:3003-3013.

10. Kikuno N, Shiina H, Urakami S, Kawamoto K, Hirata H, Tanaka Y, Place RF, Pookot D, Majid S, Igawa M, Dahiya R: Knockdown of astrocyte-elevated gene-1 inhibits prostate cancer progression through upregulation of FOXO3a activity. Oncogene 2007, 26:7647-7655.

11. Hu G, Chong RA, Yang Q, Wei Y, Blanco MA, Li F, Reiss M, Au JL, Haffty BG, Kang Y: MTDH activation by $8 \mathrm{q} 22$ genomic gain promotes chemoresistance and metastasis of poor-prognosis breast cancer. Cancer Cell 2009, 15:9-20.
12. Yu C, Chen K, Zheng H, Guo X, Jia W, Li M, Zeng M, Li J, Song L: Overexpression of astrocyte elevated gene-1 (AEG-1) is associated with esophageal squamous cell carcinoma (ESCC) progression and pathogenesis. Carcinogenesis 2009, 30:894-901.

13. Song L, Li W, Zhang H, Liao W, Dai T, Yu C, Ding X, Zhang L, Li J: Overexpression of AEG-1 significantly associates with tumour aggressiveness and poor prognosis in human non-small cell lung cancer. J Pathol 2009, 219:317-326.

14. Yoo BK, Emdad L, Su ZZ, Villanueva A, Chiang DY, Mukhopadhyay ND, Mills AS, Waxman S, Fisher RA, Llovet JM, et al: Astrocyte elevated gene-1 regulates hepatocellular carcinoma development and progression. J Clin Invest 2009, 119:465-477.

15. Jian-Bo X, Hui W, Yu-Long H, Chang-Hua Z, Long-Juan Z, Shi-Rong C, WenHua Z: Astrocyte-elevated gene-1 overexpression is associated with poor prognosis in gastric cancer. Med Oncol 2011, 28:455-462.

16. Song H, Li C, Li R, Geng J: Prognostic significance of AEG-1 expression in colorectal carcinoma. Int J Colorectal Dis 2010, 25:1201-1209.

17. Wang F, Ke ZF, Sun SJ, Chen WF, Yang SC, Li SH, Mao XP, Wang LT: Oncogenic roles of astrocyte elevated gene-1 (AEG-1) in osteosarcoma progression and prognosis. Cancer Biol Ther 2011, 12.

18. Anttila V, Stefansson H, Kallela M, Todt U, Terwindt GM, Calafato MS, Nyholt DR, Dimas AS, Freilinger T, Muller-Myhsok B, et al: Genome-wide association study of migraine implicates a common susceptibility variant on 8q22.1. Nat Genet 2010, 42:869-873.

19. Jeon HY, Choi M, Howlett EL, Vozhilla N, Yoo BK, Lloyd JA, Sarkar D, Lee SG, Fisher PB: Expression patterns of astrocyte elevated gene-1 (AEG-1) during development of the mouse embryo. Gene Expr Patterns 2010 10:361-367.

20. Khuda II, Koide N, Noman AS, Dagvadorj J, Tumurkhuu G, Naiki Y, Komatsu T, Yoshida T, Yokochi T: Astrocyte elevated gene-1 (AEG-1) is induced by lipopolysaccharide as toll-like receptor 4 (TLR4) ligand and regulates TLR4 signalling. Immunology 2009, 128:e700-706.

21. Lee SG, Su ZZ, Emdad L, Sarkar D, Fisher PB: Astrocyte elevated gene-1 (AEG-1) is a target gene of oncogenic Ha-ras requiring phosphatidylinositol 3-kinase and c-Myc. Proc Natl Acad Sci USA 2006, 103:17390-17395.

22. Liu L, Wu J, Ying Z, Chen B, Han A, Liang Y, Song L, Yuan J, Li J, Li M: Astrocyte elevated gene-1 upregulates matrix metalloproteinase- 9 and induces human glioma invasion. Cancer Res 2010, 70:3750-3759.

23. Kessenbrock K, Plaks V, Werb Z: Matrix metalloproteinases: regulators of the tumor microenvironment. Cell 2010, 141:52-67.

24. Hanahan D, Weinberg RA: Hallmarks of cancer: the next generation. Cell 2011, 144:646-674

25. Yoo BK, Gredler R, Vozhilla N, Su ZZ, Chen D, Forcier T, Shah K, Saxena U, Hansen U, Fisher PB, Sarkar D: Identification of genes conferring resistance to 5-fluorouracil. Proc Natl Acad Sci USA 2009, 106:12938-12943.

26. Yoo BK, Chen D, Su ZZ, Gredler R, Yoo J, Shah K, Fisher PB, Sarkar D: Molecular mechanism of chemoresistance by astrocyte elevated gene-1. Cancer Res 2010, 70:3249-3258.

27. Noch E, Bookland M, Khalili K: Astrocyte-elevated gene-1 (AEG-1) induction by hypoxia and glucose deprivation in glioblastoma. Cancer Biol Ther 2011, 11:32-39.

28. Bhutia SK, Kegelman TP, Das SK, Azab B, Su ZZ, Lee SG, Sarkar D, Fisher PB: Astrocyte elevated gene-1 induces protective autophagy. Proc Natl Acad Sci USA 2010, 107:22243-22248.

29. Li J, Yang L, Song L, Xiong H, Wang L, Yan X, Yuan J, Wu J, Li M: Astrocyte elevated gene- 1 is a proliferation promoter in breast cancer via suppressing transcriptional factor FOXO1. Oncogene 2009, 28:3188-3196.

30. Lee SG, Su ZZ, Emdad L, Sarkar D, Franke TF, Fisher PB: Astrocyte elevated gene-1 activates cell survival pathways through PI3K-Akt signaling. Oncogene 2008, 27:1114-1121

31. Emdad L, Lee SG, Su ZZ, Jeon HY, Boukerche H, Sarkar D, Fisher PB: Astrocyte elevated gene-1 (AEG-1) functions as an oncogene and regulates angiogenesis. Proc Natl Acad Sci USA 2009, 106:21300-21305.

32. Li C, Li R, Song H, Wang D, Feng T, Yu X, Zhao Y, Liu J, Wang Y, Geng J: Significance of aeg-1 expression in correlation with vegf, microvessel density and clinicopathological characteristics in triple-negative breast cancer. J Surg Oncol 2010.

33. Sarkar D, Park ES, Emdad L, Lee SG, Su ZZ, Fisher PB: Molecular basis of nuclear factor-kappaB activation by astrocyte elevated gene-1. Cancer Res 2008, 68:1478-1484. 
34. Glozak MA, Sengupta N, Zhang X, Seto E: Acetylation and deacetylation of non-histone proteins. Gene 2005, 363:15-23.

35. Vivanco I, Sawyers CL: The phosphatidylinositol 3-Kinase AKT pathway in human cancer. Nat Rev Cancer 2002, 2:489-501.

36. Yoo BK, Santhekadur PK, Gredler R, Chen D, Emdad L, Bhutia S, Pannell L, Fisher PB, Sarkar D: Increased RNA-induced silencing complex (RISC) activity contributes to hepatocellular carcinoma. Hepatology 2011, 53:1538-1548.

37. Blanco MA, Aleckovic M, Hua Y, Li T, Wei Y, Xu Z, Cristea IM, Kang Y: Identification of Staphylococcal Nuclease Domain-containing 1 (SND1) as a Metadherin-interacting Protein with Metastasis-promoting Functions. J Biol Chem 2011, 286:19982-19992.

38. Tong X, Drapkin R, Yalamanchili R, Mosialos G, Kieff E: The Epstein-Barr virus nuclear protein 2 acidic domain forms a complex with a novel cellular coactivator that can interact with TFIIE. Mol Cell Biol 1995, 15:4735-4744.

39. Leverson JD, Koskinen PJ, Orrico FC, Rainio EM, Jalkanen KJ, Dash AB, Eisenman RN, Ness SA: Pim-1 kinase and p100 cooperate to enhance cMyb activity. Mol Cell 1998, 2:417-425.

40. Valineva T, Yang J, Palovuori R, Silvennoinen O: The transcriptional coactivator protein p100 recruits histone acetyltransferase activity to STAT6 and mediates interaction between the CREB-binding protein and STAT6. J Biol Chem 2005, 280:14989-14996.

41. Caudy AA, Ketting RF, Hammond SM, Denli AM, Bathoorn AM, Tops BB, Silva JM, Myers MM, Hannon GJ, Plasterk RH: A micrococcal nuclease homologue in RNAi effector complexes. Nature 2003, 425:411-414.

42. Scadden AD: The RISC subunit Tudor-SN binds to hyper-edited doublestranded RNA and promotes its cleavage. Nat Struct Mol Biol 2005, 12:489-496.

43. Yoo BK, Emdad L, Lee SG, Su ZZ, Santhekadur P, Chen D, Gredler R, Fisher PB, Sarkar D: Astrocyte elevated gene-1 (AEG-1): A multifunctional regulator of normal and abnormal physiology. Pharmacol Ther 2011, 130:1-8.

doi:10.1186/2045-3701-1-36

Cite this article as: Ying et al:: Astrocyte elevated gene 1: biological functions and molecular mechanism in cancer and beyond. Cell \& Bioscience 2011 1:36.

\section{Submit your next manuscript to BioMed Central and take full advantage of:}

- Convenient online submission

- Thorough peer review

- No space constraints or color figure charges

- Immediate publication on acceptance

- Inclusion in PubMed, CAS, Scopus and Google Scholar

- Research which is freely available for redistribution

Submit your manuscript at www.biomedcentral.com/submit
C Biomed Central 